neoplasms ${ }^{3,4}$ that were excluded from the analysis performed by Cochand-Priollet et al. Most important, blinded, prospective, large-scale, multicenter clinical trials to investigate these markers are lacking. High-quality investigations are critical for any molecular analysis before findings can be translated into clinical practice. Lastly, we note an error in the letter from Pusztaszeri: the sensitivity of the gene-expression classifier was $92 \%$, not $52 \%$.

We agree with Dominguez that both negative and positive predictive values are influenced by the prevalence of cancer in a population. It is reasonable to characterize the performance of a diagnostic test with negative likelihood ratios, which is independent of the disease prevalence. However, computation of the negative likelihood ratio is accurate only when all other characteristics of the cohort are the same - a condition that is rare in the clinical setting. ${ }^{5}$ Our validation study included many sites and ethnic groups across the United States, including academic and community practices, patients ranging in age from 22 to 85 years, various nodule sizes, and many methods of fine-needle aspiration biopsy. We therefore believe our results are applicable for broad routine use.
Erik K. Alexander, M.D.

Brigham and Women's Hospital

Boston, MA

ekalexander@partners.org

Giulia C. Kennedy, Ph.D.

Veracyte

South San Francisco, CA

Bryan R. Haugen, M.D.

University of Colorado School of Medicine

Aurora, $\mathrm{CO}$

Since publication of their article, the authors report no further potential conflict of interest.

1. Duick DS, Klopper JP, Diggans JC, et al. The impact of benign gene expression classifier test results on the endocrinologist-patient decision to operate on patients with thyroid nodules with indeterminate fine-needle aspiration cytopathology. Thyroid 2012;22:996-1001.

2. Saleh HA, Feng J, Tabassum F, Al-Zohaili O, Husain M, Giorgadze T. Differential expression of galectin-3, CK19, HBME1, and Ret oncoprotein in the diagnosis of thyroid neoplasms by fine needle aspiration biopsy. Cytojournal 2009;6:18.

3. Fischer S, Asa SL. Application of immunohistochemistry to thyroid neoplasms. Arch Pathol Lab Med 2008;132:359-72.

4. de Matos PS, Ferreira AP, de Oliveira Facuri F, Assumpção LVM, Metze K, Ward LS. Usefulness of HBME-1, cytokeratin 19 and galectin-3 immunostaining in the diagnosis of thyroid malignancy. Histopathology 2005;47:391-401.

5. Pepe MS. The statistical evaluation of medical tests for classification and prediction. Oxford, England: Oxford University Press, 2003.

DOI: 10.1056/NEJMc1211421

\title{
Tofacitinib in Active Ulcerative Colitis
}

TO THE EDITOR: Sandborn et al. (Aug. 16 issue) ${ }^{1}$ report that tofacitinib has efficacy in the treatment of ulcerative colitis. The authors note that Janus kinase (JAK) inhibitors JAK1 and JAK3 are surprisingly ineffective in the treatment of Crohn's disease. ${ }^{2}$ In Crohn's disease, the major cytokines arise from the differentiation of two subtypes of CD4+ T cells - Th1 and Th17 whereas in ulcerative colitis a Th2-like differentiation process seems to be involved. Several recent reports on different experimental models seem to indicate that JAK inhibitors such as tofacitinib suppress Th2 but potentiate Th1 and Th17 responses. For example, a low dose of tofacitinib accelerates the onset of experimental autoimmune encephalomyelitis by potentiating Th17 differentiation. ${ }^{3}$ JAK inhibitors such as tofacitinib and pyridone 6 , a pan-JAK inhibitor, also strongly reduce Th2 response and potentiate Th1 and Th17 responses in different diseases. ${ }^{4}$
Moreover, tofacitinib has been also described as inhibiting interleukin-4-dependent Th2 cell differentiation in both murine and human T cells. ${ }^{5}$

Stéphane Paul, Ph.D.

Xavier Roblin, M.D.

Medical University of Saint Etienne

St. Etienne, France

xavier.roblin@chu-st-etienne.fr

No potential conflict of interest relevant to this letter was reported.

1. Sandborn WJ, Ghosh S, Panes J, et al. Tofacitinib, an oral Janus kinase inhibitor, in active ulcerative colitis. N Engl J Med 2012;367:616-24.

2. Sandborn WJ, Ghosh S, Panes J, Vranic I, Spanton J, Niczychowski W. Phase 2 randomized study of CP-690,550, an oral Janus kinase inhibitor, in active Crohn's disease. Gastroenterology 2011;140:Suppl:S124.

3. Yoshida H, Kimura A, Fukaya T, et al. Low dose CP-690,550 (tofacitinib), a pan-JAK inhibitor, accelerates the onset of experimental autoimmune encephalomyelitis by potentiating Th17 differentiation. Biochem Biophys Res Commun 2012;418:234-40.

4. Derenzini E, Lemoine M, Buglio D, et al. The JAK inhibitor AZD1480 regulates proliferation and immunity in Hodgkin lymphoma. Blood Cancer J 2011;1(12):e46. 
5. Ghoreschi K, Jesson MI, Li X, et al. Modulation of innate and adaptive immune responses by tofacitinib (CP-690,550). J Immunol 2011;186:4234-43.

DOI: $10.1056 / N E J M c 1211073$

TO THE EDITOR: In the evaluation of tofacitinib in the treatment of active ulcerative colitis by Sandborn et al., the population selection was heterogeneous in terms of previous treatment received, with the majority of patients (52 to $70 \%$ ) having received mesalamine, 27 to $58 \%$ having received glucocorticoids, and 37 to $42 \%$ having received immunosuppressants; none of these treatments had been effective. No mention is made of glucocorticoid dependence or resistance.

In clinical practice, when patients with moderate-to-severe ulcerative colitis show no improvement in response to treatment with mesalamine or a dosage of glucocorticoids lower than $30 \mathrm{mg}$ per day, guidelines indicate that the dosage of glucocorticoids may be increased to 0.75 to $1.0 \mathrm{mg}$ per kilogram of body weight, a level at which there is an acceptable rate of remission. ${ }^{1}$ In the trial conducted by Sandborn et al., the remission rate was $48 \%$ in patients receiving $10 \mathrm{mg}$ of tofacitinib twice daily, a result that is not impressive for patients in whom treatment with mesalamine or less than $30 \mathrm{mg}$ per day of glucocorticoids was not effective. Glucocorticoid withdrawal was not considered as an end point another weakness of this study. In conclusion, we do not find this new drug to be a promising novel therapeutic since the trial presented did not give clinicians a clear indication of the circumstances in which it should be used.

Mario Cottone, M.D.
University of Palermo
Palermo, Italy
dickens@tin.it
Ambrogio Orlando, M.D.
Ospedali Riuniti Villa Sofia Cervello
Palermo, Italy
Claudio Papi, M.D.
Ospedale San Filippo Neri
Rome, Italy
$\quad$ No potential conflict of interest relevant to this letter was re-
ported.

1. Travis SP, Stange EF, Lémann M, et al. European evidencebased consensus on the management of ulcerative colitis: current management. J Crohns Colitis 2008;2:24-62.

DOI: 10.1056/NEJMc1211073
THE AUTHORS REPLY: The effects of tofacitinib in patients with ulcerative colitis that we reported were from a phase 2 study. This study was designed to provide proof-of-concept for tofacitinib as an induction therapy and to elucidate the safety and efficacy profiles of various doses of tofacitinib to take forward for further investigation in larger phase 3 studies; these goals were achieved. On the basis of the study results, a phase 3 clinical program has been initiated, consisting of two studies investigating the use of tofacitinib as an induction therapy (ClinicalTrials.gov numbers, NCT01465763 and NCT01458951), a third study in which tofacitinib is used as a maintenance therapy (NCT01458574), and an open-label, longterm extension study (NCT01470612). This program should provide data that will allow clinicians to make more informed decisions about the potential use of tofacitinib in practice, including the specific patient populations likely to benefit from treatment, the effects of tofacitinib when used as maintenance therapy, and the effects when patients are spared from the use of glucocorticoids - information sought by Cottone et al.

The effects of tofacitinib in patients with Crohn's disease were studied in a separate phase 2a study. ${ }^{1}$ Although there was no evidence of significant clinical efficacy, dose-dependent reductions in levels of C-reactive protein and significant reductions in fecal levels of calprotectin were observed in the group receiving $15 \mathrm{mg}$ of tofacitinib twice a day. We acknowledge the possibility raised by Paul and Roblin that mechanistic differences in the pathophysiologies of ulcerative colitis and Crohn's disease contributed to the different outcomes seen in each study. However, neither the precise role played by JAK inhibition in the pathogenesis of inflammatory bowel disease nor the disease-specific differences in its activity is well understood, and it is also possible that the lack of observed clinical efficacy in the trial addressing Crohn's disease can be attributed to certain features of study design. An additional Phase $2 \mathrm{~b}$ clinical program, including three studies (NCT01393626, NCT01393899, and NCT01470599), has been initiated to further investigate whether the positive biomarker signals observed with tofacitinib in the previous study can be translated into clinical efficacy for patients with Crohn's disease. 
William J. Sandborn, M.D.

University of California San Diego

La Jolla, CA

wsandborn@ucsd.edu

Chinyu Su, M.D.

Wojciech Niezychowski, M.D.

Pfizer

Collegeville, PA
Since publication of their article, the authors report no further potential conflict of interest.

1. Sandborn WJ, Ghosh S, Panes J, Vranic I, Spanton J, Niezychowski W. Phase 2 randomized study of CP-690,550, an oral Janus kinase inhibitor, in active Crohn's disease. Gastroenterology 2011;140:Suppl:S124.

DOI: 10.1056/NEJMc1211073

\section{Effect of HIV-2 Infection on HIV-1 Disease Progression}

TO THE EDITOR: Esbjörnsson and colleagues (July 19 issue $)^{1}$ conclude that infection with human immunodeficiency virus type 2 (HIV-2) attenuates the course of HIV-1 infection. Their report raises three issues. First, in Guinea-Bissau, up to $30 \%$ of persons infected with both HIV-1 and HIV-2 have coinfection with human T-cell lymphotropic virus type 1 (HTLV-1), ${ }^{2}$ a circumstance that leads to an increased percentage of CD4+ T cells. ${ }^{3}$ The authors did not control for coinfection with HTLV-1, a condition that may well have contributed to the high percentage of CD4+ $\mathrm{T}$ cells among persons with dual HIV infection. Second, despite the study's long follow-up time, the authors did not treat age as a time-dependent variable in the Cox regression analyses; failure to have done so may have led to the appearance of a reduction in the rate of disease progression. In a recently published survival analysis (including 70 participants with dual HIV infection who were followed for 20 years) in which age was treated as a time-dependent variable, no differences in mortality were observed between participants with HIV-1 infection only and those with dual infection. ${ }^{4}$ Finally, none of six studies comparing mortality rates between participants with HIV-1 infection only and participants with dual infection reported lower mortality among participants with dual infection (see the figure published with the full text of this letter at NEJM.org) ${ }^{5}$; the findings of Esbjörnsson and colleagues seem to be at odds with the findings in these studies and with the fact that HIV-2 infection does not affect HIV-1 viral load in persons with dual infection. ${ }^{5}$

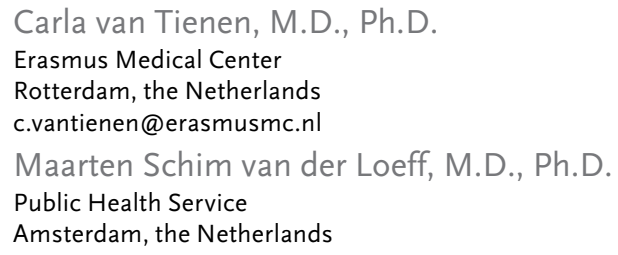

\author{
Hilton Whittle, M.D., Ph.D. \\ London School of Hygiene and Tropical Medicine \\ London, United Kingdom \\ No potential conflict of interest relevant to this letter was re- \\ ported. \\ 1. Esbjörnsson J, Mansson F, Kvist A, et al. Inhibition of HIV-1 \\ disease progression by contemporaneous HIV-2 infection. $\mathrm{N}$ Engl \\ J Med 2012;367:224-32. \\ 2. van Tienen C, Schim van der Loeff MF, Peterson I, et al. \\ HTLV-1 in rural Guinea-Bissau: prevalence, incidence and a con- \\ tinued association with HIV between 1990 and 2007. Retrovirol- \\ ogy 2010;7:50. \\ 3. Brites C, Sampalo J, Oliveira A. HIV/human T-cell lympho- \\ tropic virus coinfection revisited: impact on AIDS progression. \\ AIDS Rev 2009;11:8-16. \\ 4. van Tienen C, Schim van der Loeff M, Peterson I, et al. HTLV- \\ 1 and HIV-2 infection are associated with increased mortality in \\ a rural West African community. PLoS One 2011;6(12):e29026. \\ 5. de Silva TI, van Tienen C, Rowland-Jones SL, Cotten M. Dual \\ infection with HIV-1 and HIV-2: double trouble or destructive \\ interference? HIV Ther 2010;4:305-23.
}

DOI: $10.1056 / N E J M c 1210334$

TO THE EDITOR: Esbjörnsson and colleagues report that HIV-2 infection can favorably modulate the course of early HIV-1 infection, with reduced HIV-1 genetic diversity serving as a marker for delayed disease progression. Unraveling the mechanism behind these findings could help define immune correlates of protection from HIV-1 disease. In our view, preexisting HIV-2-specific T-cell responses in the study participants are the most likely explanation for both observations. We have previously shown that the presence of high-magnitude Gag-specific T-cell responses in persons with HIV-2 infection is inversely correlated with HIV-2 viremia. ${ }^{1}$ These responses are more polyfunctional than HIV-1-specific responses. ${ }^{2}$ HIV-1 and HIV-2 Gag have a shared sequence identity of $60 \%$. Recognition of naturally processed HIV-1 Gag antigens for alleles such as HLA-B35 and HLA-B58 (both of which are common in Guinea-Bissau) by HIV-2-specific cytotoxic T-lymphocytes (CTLs) has been reported. ${ }^{3,4}$ 